\title{
Extraction of 3D Features from Complex Environments in Visual Tracking Applications
}

\author{
Marta Marrón ${ }^{1}$, Juan Carlos García ${ }^{1}$, Miguel Ángel Sotelo ${ }^{1}$, Daniel Pizarro Pérez ${ }^{1}$, Ignacio Bravo Muñoz ${ }^{1}$ \\ ${ }^{1}$ Electronics Department. University of Alcalá, 28871, Alcalá de Henares (Madrid) SPAIN
}

\begin{abstract}
In this paper, it is presented an algorithm for processing visual data to obtain relevant information that will be afterwards used to track the different moving objects in complex indoor environments. In autonomous robots applications, visual detection of the obstacles in a dynamic environment from a mobile platform is a complicated task. The robustness of this process is fundamental in tracking and navigation reliability for autonomous robots. The solution exposed in the document is based on a stereo-vision system; so that $3 D$ information related to each object position in the local environment of the robot is extracted directly form the cameras. In the proposed application, all objects, both dynamic and static, in the local environment of the robot but the structure of the environment itself are considered to be obstacles. With this specification a distinction between building elements (ceiling, walls, columns and so on) and the rest of items in the robot surroundings is needed. Therefore, a classification has to be developed altogether with the detection task. On the other hand, the obtained data can be used to implement a partial reconstruction of the environmental structure that surrounds the robot. All these algorithms explained in detail in the following paragraphs and visual results are also included at the end of the paper.
\end{abstract}

Keywords - Stereo-vision, tracking, dynamic-crowded indoor environments.

\section{INTRODUCTION}

Visual tracking is one of the areas of greatest interest in robotics, as it is related with many topics such as visual surveillance or mobile robots navigation. Multiple approaches to this problem have been, therefore, developed by the researching community during the last decades. Among all these solutions an interesting classification can be done according to the method used to detect or extract information about the objects in the scene from the image:

1) If a static camera is used: background subtraction is generally applied to extract the image information that corresponds to dynamic objects in the scene. This method is very spread among the research community ([3], [4], [1], [2]), mainly in surveillance applications.

2) If the specific model of the object to be tracked is known: This is very common in tracking applications, both using static cameras ([4], [1], [2]) or dynamic ones ([5], [6]). The detection process is computational more expensive, but the number of false alarms and the robustness of the detector are bigger than in the case of looking for any kind of objects in the scene.

All the works referred before solve the detection problem quite easily thanks to the application of the mentioned restrictions. In the work presented in this paper none of these specifications are completed.

The solution is then, more complicated:

1) Background subtraction cannot be used, as its visual appearance changes continuously.

2) Any element in the visual environment of the robot may be an obstacle, apart from the objects that belong to the building structure in which the robot is located.

In this situation, it seems to be necessary to develop a more generic classifier that organizes visual data included in the images in two clusters:

a) Measurements coming from obstacles.

b) Measurements coming from the environment.

Once the information is classified, data assigned to cluster a) can be used as an input in any of the tracking algorithms proposed by the scientific community, such as the one designed by the authors and presented in [8] and [7]

At the same time, data classified in the environment cluster can be used to do a reconstruction of the robot surrounding structure. This last process is especially interesting for the navigation system of the robot, as the partial reconstruction of the environment can be feedback to the navigation process in order to know the exact position of the mobile robot in a local moving task, or in order to build a local map of the path travelled by the robot.

Fig. 1 shows a functional description of the global tracking application described in previous paragraphs. Some of the ideas presented in this paper have already been proven in a different application developed by the authors ([10]).IEEE Body Text

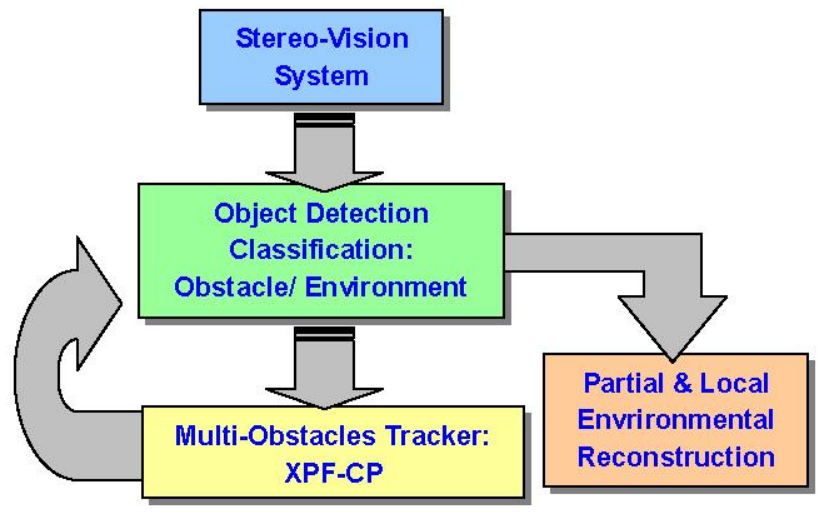

Fig. 1. General description of the global tracking application. 


\section{STEREO-VISION AND EPIPOLAR GEOMETRY}

A stereo-vision process has been thought as the most adequate system for obtaining information about the dynamic environment at each sampling time. That is because:

1) The amount of information that can be extracted from an image is much bigger than the one that can be obtained from any other kind of sensor, such as laser or ultrasound [9].

2) As the environmental configuration changes with time, the depth coordinate of the objects' position vector cannot be obtained with an only camera, and thus, a stereo-vision arrangement is needed. The acquisition system used is based on two synchronized statically arranged one next to the other.

Fig. 2 shows a functional description of the epipolar process, where the relevance of keeping constant and known extrinsic parameters $\left(\left[R_{l r}, T_{l r}\right]\right)$ and intrinsic (mainly $\left[f_{l}, f_{r}\right]$ ) ones, through a calibration of the vision system, is denoted.

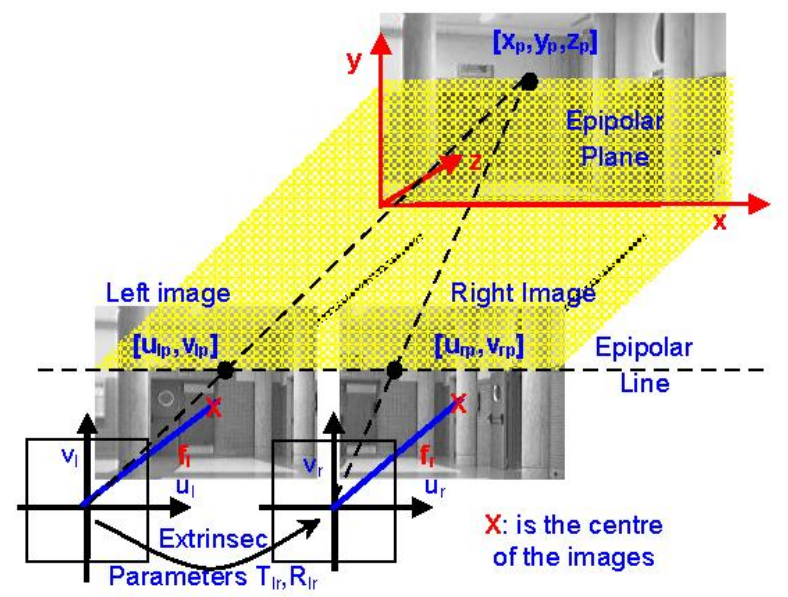

Fig. 2. Functional description of the stereo-vision data extraction process based on epipolar geometry.

Both types of parameters are used to obtain the fundamental matrix $\left(F_{l r}\right)$ that characterizes the stereo-vision arrangement in order to find matching points from the scene in the left and right frames.

The stereo-vision system used in the experiments is formed by two black and white digital cameras located in a static mounting arrangement, with a gap of $30 \mathrm{~cm}$ between them, and at a height of around $1.5 \mathrm{~m}$ from the floor. Data processing is done on general purpose computers [11]. The cameras platform has been calibrated and the intrinsic parameters for each camera, as well as the extrinsic parameters that relate them have been calculated in order to obtain the fundamental matrix $\left(F_{l r}\right)[12]$.

\section{THE DETECTION PROCESS}

Different strategies have been tested in order to achieve the best results classifying the measurement set. The solution chosen to solve the vision task previously exposed is described in this section. An alternative method is also presented after in this paper in order to make an efficiency comparison between them. Fig. 3 shows the functional flowchart of the detection process:

\section{A. The Canny Filter}

The global classification process (Fig. 3) is developed with each pair of frames (left and right) at each video frame.

Left image is used to extract the pixels that may be interesting in the detection process, and their 3D position will be obtained using the right image and the epipolar geometry.

To reduce execution time, these pixels are restricted to those extracted from the edges of the elements found in the left frame using a Canny filter [14]. Characteristics such as human heads, arms or legs, tables, doors, columns, etc. are visible and distinguishable also in quite crowded scenes and are easily extracted with the filter.

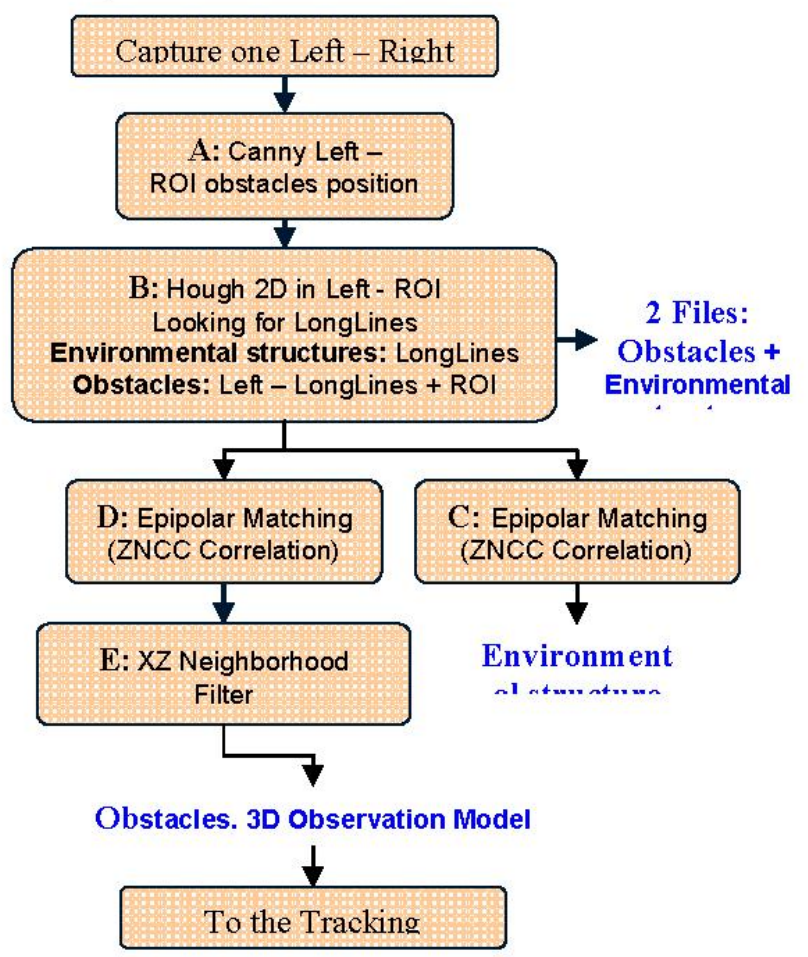

Fig. 3. Functional flowchart of the first detection and classification process designed.

Edges corresponding with environmental structures have the common characteristic of forming long lines. So, the detection process starts seeking structural shapes in the Canny image. In order to robustly find structural features in the frame, the Canny left image is zeroed in the Regions of Interest (ROIs) where an obstacle is expected to appear from the tracking algorithm results obtained in the previous time step (see Fig. 1). 


\section{B. Hough Transform}

Hough transform is then used to search long lines or long line segments in the partial Canny image. A probabilistic version of the Hough transform has been used in this application, to improve the process robustness.

\section{Epipolar Correspondence of the Line Segments classified as Structural Features}

To obtain the set of $3 \mathrm{D}$ coordinates, the correspondence problem is solved using the Zero Mean Normalized Cross Correl ation (ZNCC) because its robustness [13]

Fig. 4 shows the results obtained at the end of this step in an image extracted from a real experiment. In this image:

- Long line segments detected in the partial Canny image are shown in the upper half of the figure in different colours to distinguish them from the Canny pixels, drawn in white.

- Segments whose epipolar correspondence has been possible to find are drawn in the lower half of fig. 4 . The segments are coloured in yellow and their ending points are coloured according to the depth of its position in the 3D space (red for the farther, green for the nearer).

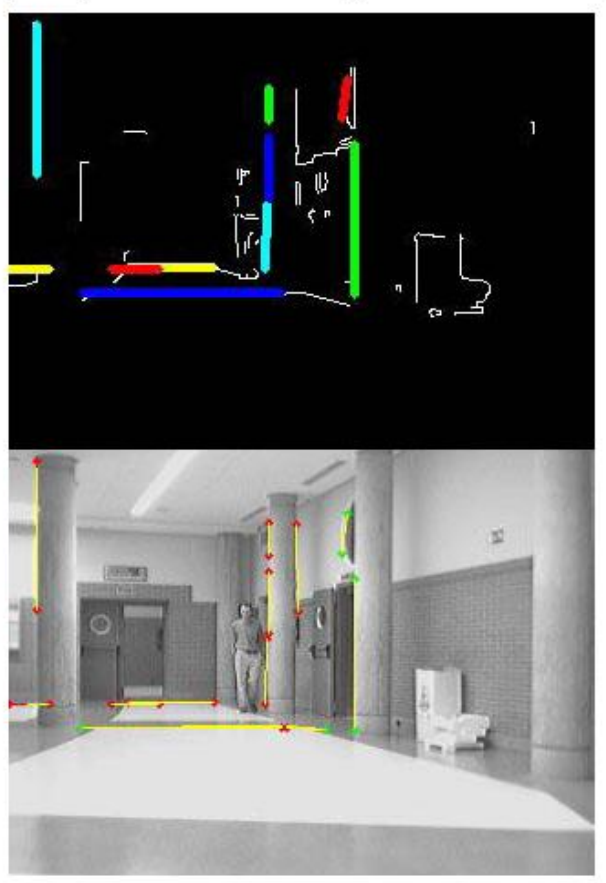

Fig. 4. Image obtained from a real experiment of the structural features detection process.

\section{Epipolar Correspondence of the Points Classified as Obstacles}

Long line segments are then erased from the full Canny image, remaining in this partial edge left image the pixels of interest in the obstacles detection process.
At the same time, regions of interest (ROIs) from the initial Canny left image that were taken out to perform the structural features detection, are set back in the edge image, this time already free of objects classified as structural ones.

Epipolar matching is then developed with these pixels, in the way described in previous paragraphs, in order to obtain its $3 \mathrm{D}$ position in the scene.

\section{E. The Neighborhood Filter}

Correspondences between points in left and right images are often not correct due to occlusions and repetitive patterns and textures, generating outliers.

In order to reject these outliers, a neighbourhood filter is developed in the $\mathrm{XZ}$ plane to all points classified in the obstacles cluster 3D space. On the other hand, the height coordinate $(\mathrm{Y})$ in each point $3 \mathrm{D}$ position vector is also used to filter the noise. So, a feasible set of points that characterizes the obstacles' position in the scene is obtained. These points will be used as the input observation model in the global tracking application (see Fig. 1 and Fig. 3).

Fig. 5 shows the results obtained at the end of this step in a real experiment.

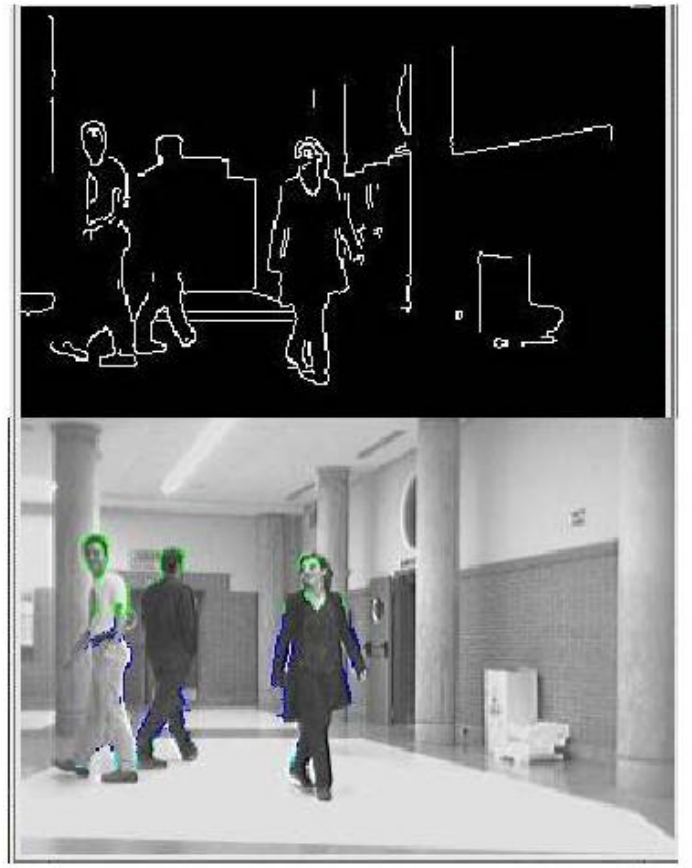

Fig. 5. Image obtained from a real experiment of the points classified as belonging to obstacles with the detection process

- Canny data points classified in the obstacle's cluster are painted in col ors in the lower image, while the upper one shows the global canny image extracted from the scene

- Colors in the lower plot indicate points' height (light blue from the floor to $0.2 \mathrm{~m}$, dark blue from $0.2 \mathrm{~m}$ to $1.5 \mathrm{~m}$ and green from $1.5 \mathrm{~m}$ to $2 \mathrm{~m}$ ).

From Fig. 5, it can be concluded that it has been achieved the objective of extracting $3 \mathrm{D}$ data that can be used in the 
pursuit tracking application, in a dynamic and complex environment.

\section{RESULTS}

The global tracking algorithm described in this paper has been implemented on a mobile 4-wheeled robot platform. Different tests have been done in unstructured indoor environments, whose results are shown in this section.

The execution time of the developed classifier is around $60 \mathrm{~ms}$, which is an acceptable solution to implement a real time acquisition process of $15 \mathrm{fps}$ to $33 \mathrm{fps}$ with images of $320 \times 240$ pixels. This sampling time depends on the number of points whose correspondence is searched, due to the ZNCC algorithm computational cost.

All vision processes have been developed using OpenCV libraries. Algorithms have been developed in a PIV at $2.2 \mathrm{GHz}$ with $512 \mathrm{Mbyes}$ of RAM.

Fig. 6 shows the classifier output in one of the real time experiments, with 5 frames of a global sequence. Classification results for each frame are displayed in 3 images organized vertically:

- The one on the top shows the edge image directly obtained applying the Canny filter to the left frame. Obstacles and environmental structure borders are mixed in this image.

- The one in the middle shows the result of the classification process in a $\mathrm{XZ}$ projection of the resulting $3 \mathrm{D}$ points ( $\mathrm{x}$ range is from -5 to $5 \mathrm{~m}$; $\mathrm{z}$ range is from 0.2 to $20 \mathrm{~m}$ ). White dots represent points classified in the obstacles cluster.

- The bottom one shows the final left image in which points classified in the obstacles cluster are highlighted with a colour according to their height in the $3 \mathrm{D}$ space (same code used in Fig. 6).

As depicted in this figure, the obstacles in the resulting 3D space are represented by a uniformly distributed set of points. It can be concluded that the classification objective has been achieved, and that the resulting obstacle data set can be used in the tracker shown in Fig. 1.

Fig. 7 shows some other results of the detection and classification algorithm presented in this paper. In this figure, there are also 5 frames extracted from a real time experiment:

- At the upper image, long line segments found by the detection process parallel to the robot walking plane are painted in green over the Canny left image.

- At the lower image, points included in the lines marked in the upper image, whose 3D position has been possible to obtain through the epipolar matching, are painted in green

As it can be noticed in both figures (6 and 7), the algorithm classifies in a very robust way features that are part of the building structure from those that are part of static or dynamic obstacles. The classification process is also robustly developed in crowded situations as the one shown in Fig. 7.

Neither the information related to the environmental structure nor the one related to the obstacles in it is processed by the application presented in this paper. A tracking algorithm as the one developed by the authors in [8], and a reconstruction algorithm still under development, are necessary to achieve the global objective presented in Fig. 1.

\section{CONCLUSIONS}

In this paper, an algorithm for detection and classification visual features in an indoor and complex environment is presented.

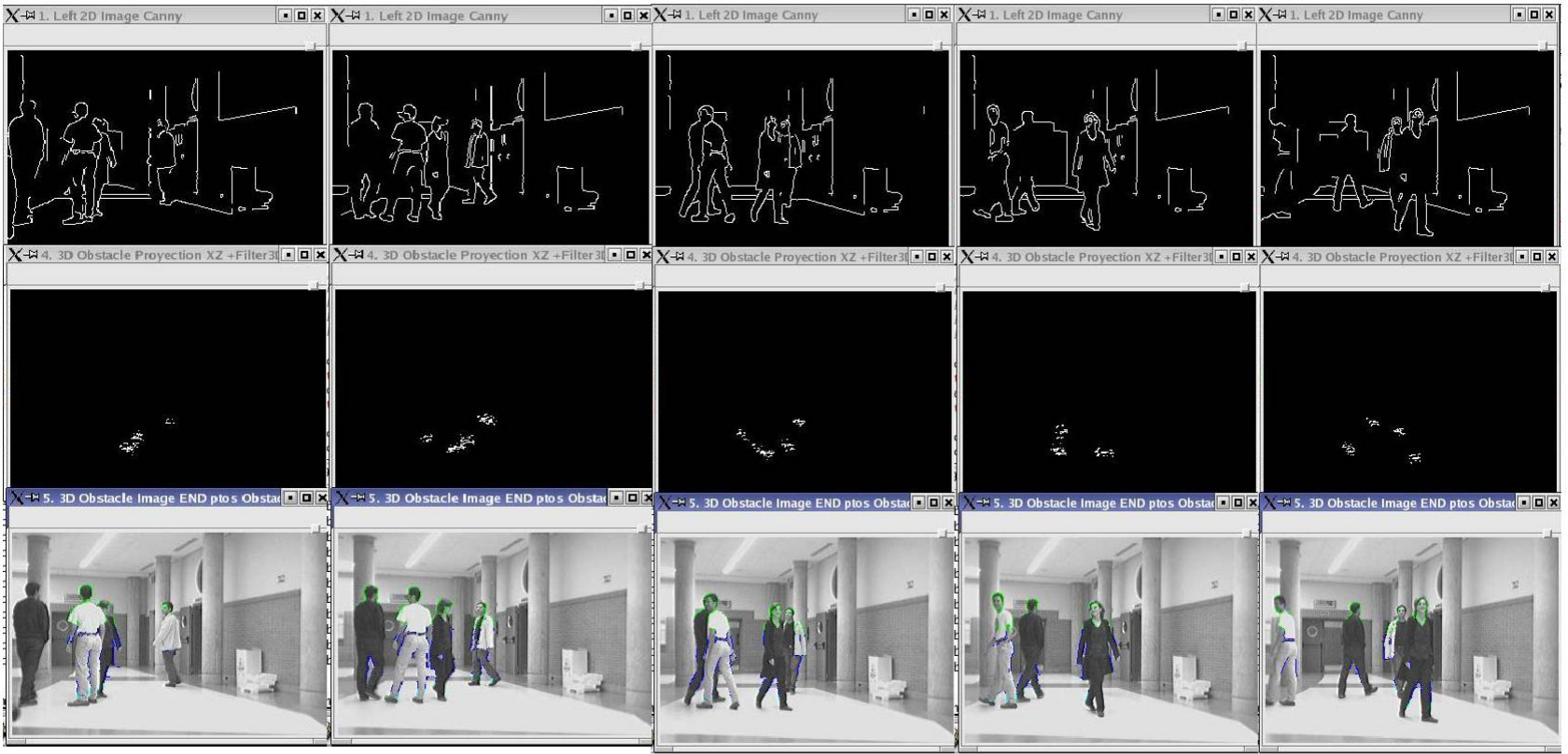

Fig. 6. Results of the classification algorithm in a real situation. Points classified in the obstacles cluster are shown in the images. 


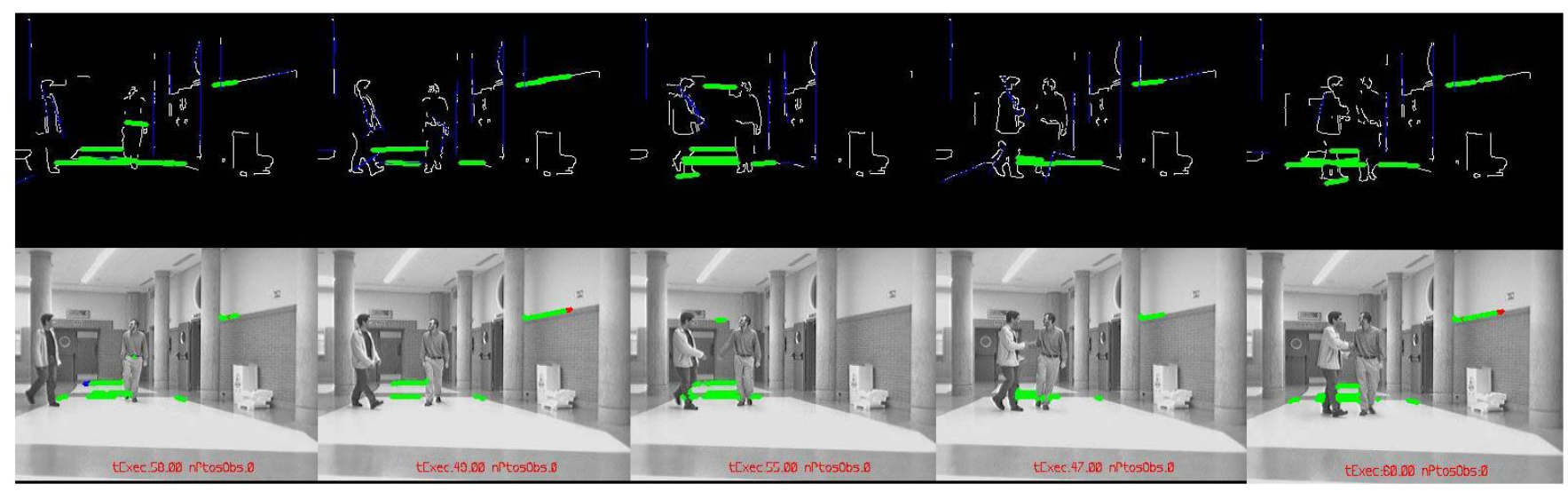

Fig. 7. Results of the classification algorithm in a real situation. Points classified in the structure cluster are shown in the images.

The detection and classification process is based on a synchronized stereo-vision system, and different vision techniques have been tested and validated in order to achieve the image process proposed.

The vision system has proven to be robust in different scenes and distances up to $20 \mathrm{~m}$.

As a future work, a pant-tilt unit can be added to the stereo-vision arrangement, in order to orientate the visualfield of the cameras to the most interesting area for the robot movement.

Results obtained with the proposed algorithm are shown in the figures included in the document, and prove that the objectives exposed have been achieved robustly and efficiently. The reliability of these results is especially important as they are thought to be used in tracking applications for robot autonomous navigation.

\section{ACKNOWLEDGMENTS}

This work was supported by the Ministry of Science and Technology under RESELAI project (reference TIN200614896-C02-01).

\section{REFERENCES}

[1] M. Isard, A. Blake. "Icondensation: Unifying low-level and high-level tracking in a stochastic framework", Proceedings of the Fifth European Conference on Computer Vision (ECCV98), Vol. 1, pp. 893-908, 1998.

[2] Y. Chen, T.S. Huang, Y. Rui. "Mode-based multi-hypothesis head tracking using parametric contours", Proceedings of the Fifth IEEE International Conference on Automatic Face and Gesture Recognition (FGR02), ISBN: 0-7695-1602-5, Washington, May 2002.

[3] Z. Khan, T. Balch, F. Dellaert. "A Rao-Blackwellized Particle Filter for Eigen Tracking", Proceedings of the Third IEEE Conference on Computer Vision and Pattern Recognition (CVPR04), pp. 980-986, Washington, June 2004.

[4] P. Torma, C. Szepesvári. "Sequential importance sampling for visual tracking reconsidered", Proceedings of the Ninth International Workshop on Artificial Intelligence and Statistics, ISBN: 0-9727358-01 , Key West, January 2003.

[5] J.M. Odobez, D. Gatica-Perez. "Embedding motion model-based stochastic tracking", Proceedings of the Seventeenth International Conference on Pattern Recognition (ICPR04), Vol. 2, pp. 815-818, Cambridge, August 2004
[6] K. Okuma, A. Taleghani, N. De Freitas, J.J. Little, D.G. Lowe. "A boosted particle filter: multi-target detection and tracking", Proceedings of the Eighth European Conference on Computer Vision (ECCV04), Lecture Notes in Computer Science, ISBN: 3-540-21984-6, Vol. 3021 Part I, pp. 28-39 Prague, May 2004.

[7] M. Marrón, J.C. García, M.A. Sotelo, E.J. Bueno. "Clustering methods for $3 \mathrm{D}$ vision data and its application in a probabilistic estimator for tracking multiple objects", Proceedings of the Thirty-First Annual Conference of the IEEE Industrial Electronics Society (IECON05), ISBN: 0-7803-9252-3, pp. 2017-2022, Raleigh, November 2005.

[8] M. Marrón, J.C. García, M.A. Sotelo, D. Fernandez, D. Pizarro. "XPFCP: An extended particle filter for tracking multiple and dynamic objects in complex environments", Proceedings of the IEEE International Symposium on Industrial Electronics 2005 (ISIE05), ISBN: 0-7803-8738-4. Vol. I-IV, pp. 1587-1593, Dubrovnik, June 2005 .

[9] M. Marron, M.A. Sotelo, J.C. García. "Design and applications of an extended particle filter with a pre-clustering process, XPFCP", Proceedings of the IEEE Conference on Mechatronics \& Robotics 2004 (MECHROB04), ISBN: 3-938153-50-X, Vol. 2/4, pp. 187-191, Aachen, September 2004.

[10] I. Parra, D. Fernández, M.A. Sotelo, P. Revenga, L.M. Bergasa, M. Ocafía, J. Nuevo, R. Flores. "Pedestrian recognition in road sequences", Proceedings of the Fifth WSEAS International Conference on Signal Processing, Robotics and Automation (ISPRA06), ISBN: 960-8457-416, pp: 273-278, Madrid, February 2006.

[11] D.M.Gavrila, V. Philomin. "Real-time object detection for smart vehicles", Proceedings of the Seventh IEEE International Conference on Computer Vision (ICCV99), Vol. 1, pp. 87-93, Corfu, September 1999.

[12] G. Xu, Z. Zhang. Epipolar Geometry in Stereo, Motion and Object Recognition: A Unified Approach. 1st ed. Kluwer Academic Publishers, London 1996.

[13] B. Boufama. Reconstruction tridimensionnelle en vision par ordinateur: Cas des cameras non etalonnees. PhD Thesis, INP de Grenoble, 1994.

[14] F.J. Canny. "A computational approach to edge detection", IEEE Transactions on Pattern Analysis and Machine Intelligence, Vol. 8, $\mathrm{n}^{\circ}$ 6, pp: 679-698, November, 1986. 